\title{
Observation of Brillouin gain spectrum in optical fibers in telecommunication band: Effect of pump wavelength
}

\author{
Heeyoung Lee ${ }^{a}$, Neisei Hayashi, Yosuke Mizuno, \\ and Kentaro Nakamura \\ Precision and Intelligence Laboratory, Tokyo Institute of Technology, \\ Yokohama 226-8503, Japan \\ a)hylee@sonic.pi.titech.ac.jp
}

Abstract: We study the optical wavelength dependence of the Brillouin gain spectrum for a silica single-mode fiber in the range from 1511 to $1555 \mathrm{~nm}$ using a measurement setup containing an erbium-doped fiber amplifier (EDFA) in the Brillouin pump path. The Brillouin peak power and linewidth are clearly dependent on the pump wavelength, the trends of which correspond well with the wavelength dependence of the EDFA gain. This result suggests that we need to consider the unique wavelength dependence of the EDFA gain to practically obtain a higher Brillouin peak power and narrower linewidth at the telecommunication wavelength.

Keywords: Brillouin scattering, optical fibers, erbium-doped fiber amplifiers

Classification: Fiber optics, Microwave photonics, Optical interconnection, Photonic signal processing, Photonic integration and systems

\section{References}

[1] T. Horiguchi and M. Tateda: J. Lightwave Technol. 7 (1989) 1170. DOI: $10.1109 / 50.32378$

[2] T. Kurashima, T. Horiguchi, H. Izumita, S. Furukawa and Y. Koyamada: IEICE Trans. Commun. E76-B (1993) 382.

[3] D. Garus, K. Krebber and F. Schliep: Opt. Lett. 21 (1996) 1402. DOI:10.1364/ OL.21.001402

[4] K. Hotate and T. Hasegawa: IEICE Trans. Electron. E83-C (2000) 405.

[5] Y. Mizuno, W. Zou, Z. He and K. Hotate: Opt. Express 16 (2008) 12148. DOI:10.1364/OE.16.012148

[6] G. P. Agrawal: Nonlinear Fiber Optics (Academic Press, Boston, 1995).

[7] A. Bergman, L. Yaron, T. Langer and M. Tur: J. Lightwave Technol. 33 (2015) 2611. DOI:10.1109/JLT.2014.2371473

[8] Z. Yang, X. Hong, J. Wu, H. Guo and J. Lin: Appl. Phys. Express 6 (2013) 072502. DOI:10.7567/APEX.6.072502

[9] S. Todoroki: Fiber Fuse: Light-induced Continuous Breakdown of Silica Glass Optical Fiber, NIMS Monographs (Springer Japan, 2014). 
[11] A. Yeniay, J. M. Delavaux and J. Toulouse: J. Lightwave Technol. 20 (2002) 1425. DOI:10.1109/JLT.2002.800291

[12] A. W. Brown, B. G. Colpitts and K. Brown: J. Lightwave Technol. 25 (2007) 381. DOI:10.1109/JLT.2006.886672

[13] Y. Mizuno, P. Lenke, K. Krebber and K. Nakamura: IEEE Photonics Technol. Lett. 24 (2012) 1496. DOI:10.1109/LPT.2012.2206803

[14] J. Kani and M. Jinno: Electron. Lett. 35 (1999) 1004. DOI:10.1049/el: 19990708

[15] L. Wei, W. Xue, Y. Chen, T. T. Alkeskjold and A. Bjarklev: Opt. Lett. 34 (2009) 2757. DOI:10.1364/OL.34.002757

[16] S. Yamamoto, K. Utaka, S. Akiba, K. Sakai, Y. Matsushima, S. Sakaguchi and N. Seki: Electron. Lett. 18 (1982) 239. DOI:10.1049/el:19820162

[17] W. Shi, E. B. Petersen, Z. Yao, D. T. Nguyen, J. Zong, M. A. Stephen, A. C. Pirson and N. Peyghambarian: Opt. Lett. 35 (2010) 2418. DOI:10.1364/OL.35. 002418

[18] E. Desurvire: Erbium-Doped Fiber Amplifiers: Principles and Applications (Wiley, New York, 1994).

[19] Y. Zhang, X. Liu, J. Peng, X. Feng and W. Zhang: IEEE Photonics Technol. Lett. 14 (2002) 290. DOI:10.1109/68.986789

[20] M. Ding, Y. Mizuno and K. Nakamura: Opt. Express 22 (2014) 24706. DOI: 10.1364/OE.22.024706

[21] Y. Mizuno and K. Nakamura: Appl. Phys. Lett. 97 (2010) 021103. DOI: $10.1063 / 1.3463038$

\section{Introduction}

Over the past few decades, optical fiber sensors based on Brillouin scattering have been extensively studied owing to their ability to measure strain and temperature distribution $[1,2,3,4,5]$. Brillouin scattering is known to be one of the most significant nonlinear phenomena in optical fibers [6]. Higher-power pump light induces even higher-power scattered light, leading to a high signal-to-noise ratio (SNR) of the sensing system [7, 8, 9, 10]. In addition, such higher-power pump light is reported to provide narrower Brillouin linewidth, resulting in a high precision of the measurement [11]. Therefore, Brillouin sensors have been mainly implemented using light sources at the telecommunication wavelength, where relatively inexpensive erbium-doped fiber amplifiers (EDFAs) have been used, though some exceptions have been reported $[12,13]$.

The typical wavelengths of laser diodes at the telecommunication wavelength are 1510,1530 , and $1550 \mathrm{~nm}[14,15,16,17]$. To date, no reports have been provided as to which wavelength is the most suitable for Brillouin observation, because the Brillouin gain spectrum (BGS) is known not to be largely dependent on the wavelength of the pump light in this range [6]. However, the BGS observed by using a practical setup containing an EDFA for preparing the Brillouin pump light should exhibit optical wavelength dependence if we consider the wavelength dependence of the gain of the EDFA [18, 19, 20].

In this work, we investigate the optical wavelength dependence of the BGS in a silica single-mode fiber (SMF) using an experimental setup containing an EDFA in the Brillouin pump path. The driving current of the 980-nm pump laser in the 
EDFA is constant. A tunable laser at $1530-1555 \mathrm{~nm}$ and a wavelength-fixed laser at $1511 \mathrm{~nm}$ are used. The Brillouin peak power and linewidth are found to exhibit large dependences on the pump wavelength, the trends of which are in good agreement with the wavelength dependence of the EDFA gain.

\section{Principle}

The pump light injected into an optical fiber interacts with acoustic phonons and generates backscattered light with a frequency downshift. This phenomenon is termed Brillouin scattering, and the scattered light spectrum is the BGS. The amount of the frequency downshift, referred to as the Brillouin frequency shift (BFS) $v_{B}$, is given by [6]

$$
v_{B}=\frac{2 n v_{a}}{\lambda_{p}},
$$

where $n$ is the refractive index, $v_{\mathrm{a}}$ is the acoustic velocity, and $\lambda_{\mathrm{p}}$ is the pump wavelength. The BFS value is known to be $\sim 11 \mathrm{GHz}$ in silica SMFs at a pump wavelength of $1550 \mathrm{~nm}$. In principle, the peak power of the BGS is influenced by the Brillouin gain coefficient $g_{\mathrm{B}}$, which is given by [6]

$$
g_{B}=\frac{2 \pi n^{7} p_{12}^{2}}{c \lambda p^{2} \rho v_{a} \Delta v_{B}},
$$

where $p_{12}$ is the longitudinal elasto-optic coefficient, $c$ is the velocity of light in vacuum, $\rho$ is the density of the fiber core, and $\Delta v_{B}$ is the Brillouin linewidth. In silica SMFs, $g_{\mathrm{B}}$ is reported to be $3-5 \times 10^{-11} \mathrm{~m} / \mathrm{W}$. When $\lambda_{\mathrm{p}}$ varies from $1511 \mathrm{~nm}$ to $1555 \mathrm{~nm}$, the change in $g_{\mathrm{B}}$ is negligible (as shown below).

The simplest method for obtaining higher-power Brillouin-scattered light is by increasing the power of the pump light, which can be easily done at the telecommunication wavelength by amplifying the pump light using an EDFA. Fluorescence in an EDFA occurs through the radiative transition of erbium ions between different energy levels - specifically, from ${ }^{4} I_{13 / 2}$ to ${ }^{4} I_{15 / 2}$ in a $980-\mathrm{nm}$ pumping configuration - and exhibits a clear wavelength dependence (note that different EDFAs exhibit different wavelength dependences of the gain) [18]. Consequently, when an EDFA is used in the pump path, the Brillouin peak power is significantly dependent on the wavelength.

\section{Experimental setup}

We employed a 1-km-long silica SMF (Brillouin threshold $=17 \mathrm{dBm}[6]$ ) as a fiber under test (FUT). The experimental setup shown in Fig. 1, which is basically the same as that previously reported [21], enabled us to observe the BGS with a high frequency resolution using self-heterodyne detection. As light sources, we employed a tunable laser at $1530-1555 \mathrm{~nm}$ and a wavelength-fixed laser at $1511 \mathrm{~nm}$, the output powers of which were set to $6 \mathrm{dBm}(=4 \mathrm{~mW})$. The linewidth of the two lasers were lower than $1 \mathrm{MHz}$. An EDFA, which operated in an automatic-currentcontrol mode, was inserted in the Brillouin pump path; the reference light and the Brillouin-scattered light were not amplified. The electrical signal was also not amplified before it was monitored using an electrical spectrum analyzer (ESA). The 
end of the FUT was cut at an angle to suppress the Fresnel reflection. The polarization state was averaged using a polarization scrambler inserted in the reference path. The room temperature was maintained at $22^{\circ} \mathrm{C}$.

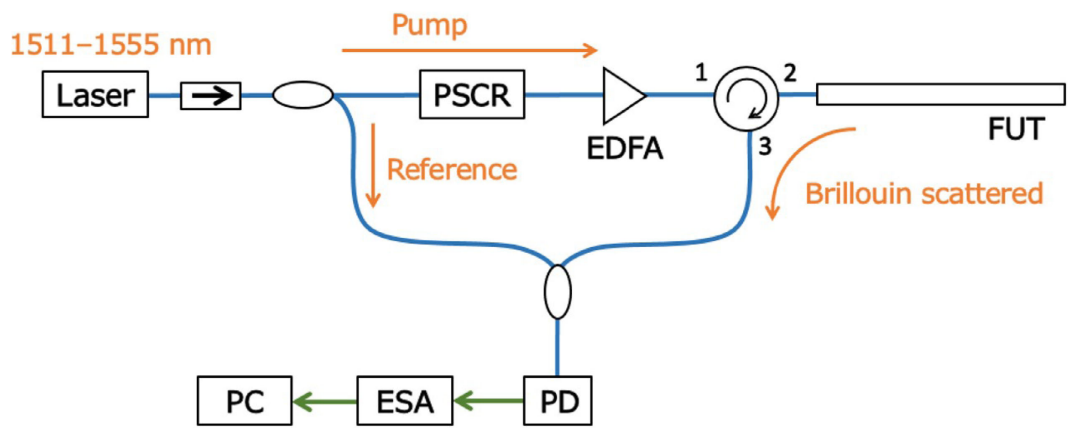

Fig. 1. Experimental setup for observing the BGS. EDFA, erbiumdoped fiber amplifier; ESA, electrical spectrum analyzer; FUT, fiber under test; PC, personal computer; PD, photodiode; PSCR, polarization scrambler

\section{Experimental results}

We verified that the laser output power and the photodiode (PD) sensitivity do not largely depend on the optical wavelength. Fig. 2(a) shows the output spectra of the two lasers. The peak power was almost the same irrespective of the wavelength. The spectral floor of the 1511-nm laser was not flat, but had no quantifiable influence on the Brillouin measurement because the maximum power of the floor was more than $40 \mathrm{~dB}$ lower than the peak power. Fig. 2(b) shows the measured BGSs corresponding to the laser outputs at each wavelength in Fig. 2(a).

The EDFA in the pump path was removed in this measurement so that this result can be interpreted as the wavelength dependence of the PD sensitivity. The PD sensitivity had a slight wavelength dependence $(0.3-\mathrm{dB}$ change in the power

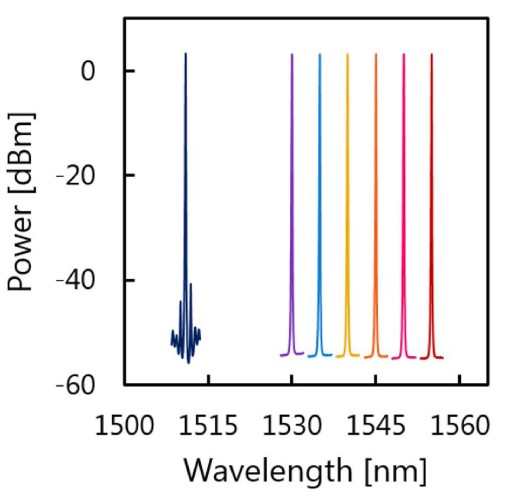

(a)

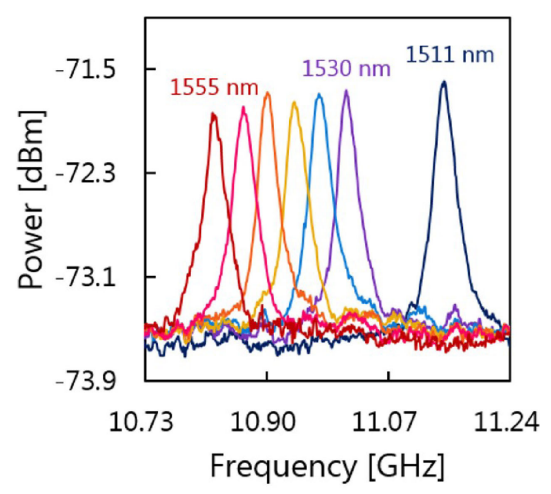

(b) 


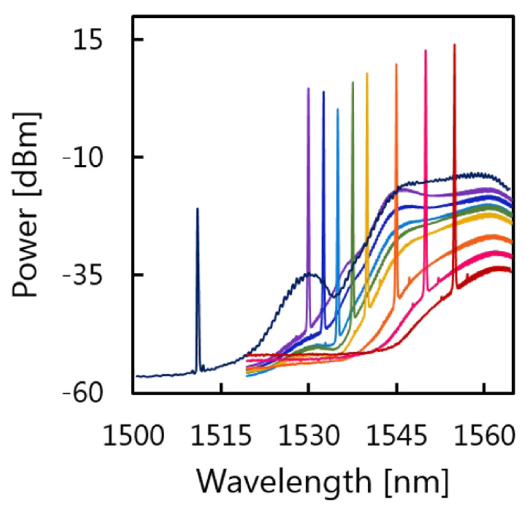

Fig. 3. Output spectra from the EDFA measured at laser wavelengths from 1511 to $1555 \mathrm{~nm}$.

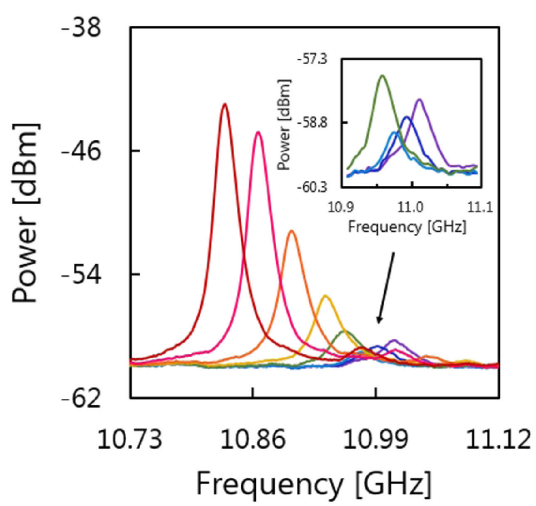

(a)

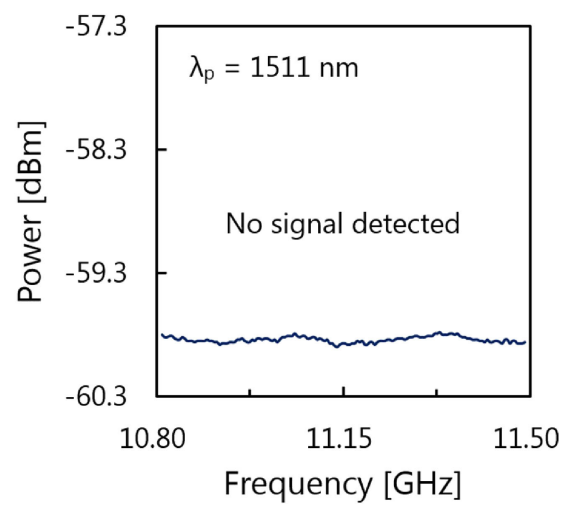

(b)

Fig. 4. (a) BGSs measured at laser wavelengths from 1530 to $1555 \mathrm{~nm}$. The inset shows a magnified view of the dip at $\sim 11.0 \mathrm{GHz}$. (b) BGS measured at $1511 \mathrm{~nm}$; no signal was detected.

in the wavelength range), which was negligible compared to the wavelength dependence of the EDFA gain discussed below.

We then measured the output spectra from the EDFA with varying wavelengths of the lasers (Fig. 3). The driving current of the 980-nm pump laser in the EDFA was set to $700 \mathrm{~mA}$, corresponding to an output of $\sim 13 \mathrm{dBm}$ at $1550 \mathrm{~nm}$; note that this value is lower than the Brillouin threshold of $\sim 17 \mathrm{dBm}$. Each laser output spectrum was amplified on the amplified spontaneous emission (ASE) spectrum [18]. The peak power of the laser output was dependent on the optical wavelength; with increasing wavelength from 1511 to $1555 \mathrm{~nm}$, the peak power increased, but a dip was observed at $1535 \mathrm{~nm}$.

Subsequently, we observed the BGSs when the laser wavelength was changed from 1530 to $1555 \mathrm{~nm}$ (Fig. 4(a)). The BGS, which should appear at $11.15 \mathrm{GHz}$ at a wavelength of $1511 \mathrm{~nm}$ according to Eq. (1), was not observed because it was hidden by the noise floor (Fig. 4(b)). As the wavelength increased, the BFS shifted to a lower frequency, which is natural according to Eq. (1). With increasing wavelength, the Brillouin peak power increased; such a clear wavelength dependence ( $>15-\mathrm{dB}$ change in power in the wavelength range) was not observed for the PD sensitivity (Fig. 2(b)). A small but clear dip was observed at $1535 \mathrm{~nm}$, 


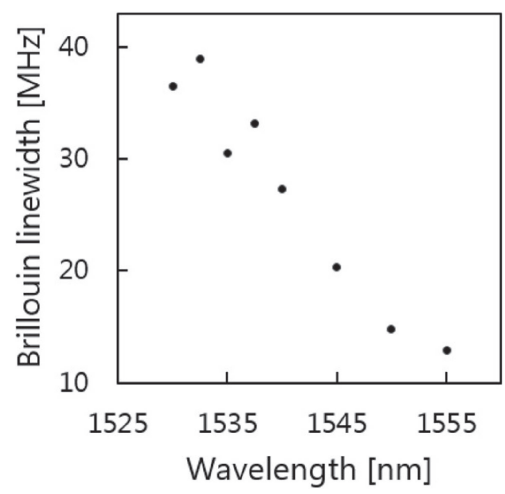

Fig. 5. Brillouin linewidth calculated at laser wavelengths from 1530 to $1555 \mathrm{~nm}$. Note that the laser wavelength is inversely proportional to the BFS.

which agrees well with the wavelength of the EDFA output at which the dip occurred. The Brillouin peak power did not exhibit a completely linear dependence on the peak power of the EDFA output, because the Brillouin peak power exponentially depends on the pump power even in the range lower than the Brillouin threshold [6].

Finally, the Brillouin linewidth dependence on the laser wavelength in the range from 1530 to $1555 \mathrm{~nm}$ was calculated using Fig. 4(a). After converting the vertical axis into a linear scale, each BGS was fitted to a Lorentzian function, the linewidth of which was defined as the Brillouin linewidth in this calculation. This definition is valid because the linewidth of the tunable laser was lower than $1 \mathrm{MHz}$ [11]. The result shown in Fig. 5 indicates that the Brillouin linewidth becomes narrower as the laser wavelength increases; this trend agrees well with the Brillouin linewidth narrowing effect detailed in Ref. [11]. The instability of the calculated values at $\sim 1535 \mathrm{~nm}$ (corresponding to the BFS of $\sim 11.0 \mathrm{GHz}$ ) may originate from the decrease in the Brillouin peak power, but the BGSs at $\sim 1535 \mathrm{~nm}$ were relatively so close to the noise floor that it was difficult to calculate the Brillouin linewidths with a sufficiently high accuracy.

\section{Conclusion}

The optical wavelength dependence of the BGS in a silica SMF was investigated in the wavelength range from 1511 to $1555 \mathrm{~nm}$ using an experimental setup containing an EDFA in the Brillouin pump path. The Brillouin peak power and linewidth exhibited a clear dependence on the pump wavelength, the trend of which corresponded well with the wavelength dependence of the EDFA gain. Thus, we showed that, to practically obtain a higher Brillouin peak power and narrower linewidth at the telecommunication wavelength, the unique wavelength dependence of the gain of the EDFA used in the system should be carefully considered. We anticipate that this result will be a practically useful guideline for selecting suitable light sources for developing strain and temperature sensors based on Brillouin scattering with a high SNR and high precision. 


\section{Acknowledgments}

This work was supported by JSPS KAKENHI Grant Numbers 25709032, 26630180, and 25007652, and by research grants from the Iwatani Naoji Foundation, the SCAT Foundation, and the Konica Minolta Science and Technology Foundation. 